\title{
Some Thoughts on Modelling Hail Impact on Surfaces
}

\author{
J. R. Macdonald ${ }^{1}$ (i) $\cdot$ M. M. Stack ${ }^{1}$
}

Received: 5 November 2020 / Revised: 5 November 2020 / Accepted: 2 December 2020 / Published online: 27 January 2021

(c) The Author(s) 2021

\begin{abstract}
Hail impact-induced erosion has the potential to significantly affect the operational lifetime of structures exposed to extreme weathering environments such as hail events. Computational materials modelling can be used to better understand the erosion behaviour during a hailstone impact, and here the relevant background work is detailed. In this paper, an implementation of an ice impact model, utilising Smooth Particle Hydrodynamics along with a highly strain-rate-dependent material model, is shown, and its results and limitations discussed. An overview is given on the literature on modelling hail events, including the history of experimental work. The various potential modelling methods which have been developed is then given, along with an evaluation of the suitability of the methods to future work in this area.
\end{abstract}

Keywords Hail $\cdot$ Erosion $\cdot$ Models $\cdot$ Simulation $\cdot$ Windfarm $\cdot$ Weathering

\section{Introduction}

Energy conversion structures in the renewable area represent a rapidly growing area of technological research, with environmental concerns giving rise to a need for economically feasible clean forms of energy generation. One particular area in need of further research is the range of factors affecting the operational lifetime of wind turbines in various environmental conditions. A key factor in this lifetime is the erosion of the leading edge of the turbine blade [1], which results in a dramatic decrease in the aerodynamic effectiveness of the blade [2] — producing less lift and more dragand hence causing a significant decrease in the power output of the turbine [3]. It is therefore of great interest to develop methods by which this leading edge erosion can be predicted and explained in terms of its modes and mechanisms, in order to predict and potentially mitigate loss in turbine output over time.

Previous research has investigated the role in rain in leading edge erosion [4], but limited work has gone towards that of hailstone impact. Hail is potentially a common occurrence in locations that possess high wind resource and thus would

J. R. Macdonald

john.r.macdonald@strath.ac.uk

1 Department of Mechanical and Aerospace Engineering, University of Strathclyde, James Weir Building, 75 Montrose Street, Glasgow G1 1XJ, UK be ideal sites for wind farm developments, and thus hail impact could form an important factor in the erosion of turbines at sites with a high frequency of hailstorms. Our aim is to characterise the role of hail in leading edge erosion by using computational modelling to simulate the effect of hailstone impact on materials commonly used in the construction of wind turbine blades, in order to better understand the threat of hail to the lifetime operation of wind turbines in a variety of environmental conditions.

\subsection{Hail Impact Experimental Literature}

A significant early experimental investigation into ice impact on fibre-reinforced composite materials was carried out by Kim and Kedward [5], who created "simulated hail ice" (SHI) by freezing water inside a spherical mould. Two different constructions of SHI were used in the experiments'monolithic' spheres made by filling the entire mould at once, and 'flatwise layered' spheres made by alternately filling and freezing water in the mould over 10-11 sessions, in an attempt to more accurately approximate real hailstones. The SHI was then fired using a Nitrogen gas cannon apparatus at both a Force Transducer and a carbon fibre composite panel.

The force transducer measurements showed that during impacts the contact force quickly reaches a peak before quickly decaying away-with the entire process taking approximately $1 \mathrm{~ms}$, with the peak force taking a value 
between 4 and $90 \mathrm{kN}$ depending approximately linearly on the diameter of the SHI, and also on the construction-with layered ice giving rise to larger peak forces for larger diameters of SHI. The impact tests on composite panels aimed to find the failure threshold energy (FTE) - the kinetic energy value at which the panel begins to show signs of damagefor various panel configurations and ice sphere sizes. It was found that the failure threshold energy is proportional to the square of the sphere diameter and also linearly with the thickness of the composite panel. For lower speed impacts (just above the failure threshold energy) delamination was the primary mode of failure seen, while higher speed impacts resulted in fibre failure and cracks through the material. While the experimental apparatus used included a highspeed camera setup, the quality of the image was insufficient to see the behaviour of the SHI sphere during impact in any detail. Better high-speed footage of SHI impact was captured during the experimental work of Tippmann [6], and in the provided frame it can be seen that the ice breaks up significantly during impact as cracks propagate backwards through the projectile from the point of contact, shattering the ice into many smaller particles.

\subsection{Hail Impact Modelling Literature}

As well as their experimental investigation, Kim and Kedward [5] also provided one of the first major computational models of hail impact, using the explicit finite element package DYNA3D, which would later become LS-DYNA. The ice projectile model utilised a Lagrangian FEA approach with a material model that allowed for the propagation of microcracks through the projectile. Only the case of a monolithic SHI sphere was considered in this modelling work, and thus the model did not attempt to model the effect of structure on the projectile response to the impact. In the initial model of the force transducer experiment, the numerically calculated peak impact force showed similar values with the same dependence on SHI diameter as in the experimental results for monolithic SHI.

Once the ice projectile model had been created and validated, it was then used to simulate impact on a composite panel as in the second part of the experimental work. This model found that Interlaminar shear stress is the first mechanism of failure to reach significant levels, concurring with the experimental observation of delamination in lowervelocity impacts and indicating that delamination is the primary mode of material failure in composites, particularly in impacts with the lower-diameter categories of hailstone.

Later Carney et al. [7] would expand upon this work by developing a material model for ice that took into account an important physical property of ice-i.e. its behaviour under compression is highly sensitive to strain rate [8]. When compressed slowly ice is ductile, but when rapidly compressed it instead displays brittle behaviour, yielding very little before failure. The transition between these states happens at a strain rate of approximately $10^{-4}$ to $10^{-3} \mathrm{~s}^{-1}$, depending on the grain size of the specimen in question. This is an important consideration when modelling high-speed impacts as very high strain rates are observed in such scenarios, and the ice material behaviour during impact affects how the impact energy dissipates through the projectile, which affects the peak force and thus the potential damage to the target. It is therefore imperative that this behaviour is accurately reflected in the ice material model. Also used in this treatment was an Eulerian approach to model the projectile, as opposed to the Lagrangian models used previously-this change in method better deals with the large deformations in the ice sphere during the impact event.

Anghileri et al. [9] conducted a comparative study of three different modelling methods of spherical SHILagrangian Finite Element, Arbitrary Lagrangian Eulerian, and Smooth Particle Hydrodynamics. After validating the three models with experimental data, each was used to simulate the same impact scenario in order to provide a direct comparison between the models. The Lagrangian FE model was found to be insufficient in this case as the mesh distortion becomes too large. The SPH formulation was found to be the most appropriate for use, both in terms of numerical accuracy and computational time, with the SPH simulations taking just over an hour to complete while the FE and ALE models took 39 and $14 \mathrm{~h}$, respectively. Juntikka and Olsson [10] would later apply the SPH approach to a spherical projectile, using a material model similar to Kim and Kedward [5], showing that the physical behaviour reproduced that seen in experiment closely, with a stress wave passing through the material and causing cracking into many smaller particles.

Keegan [11] sought to use the SPH methodology with the Carney strain-rate-dependent material model in application to the wind turbine blade erosion problem, but the validity of the combined model had yet to be established. This model was validated by simulating the same experimental setup as Carney, but switching the Eulerian approach with SPH. This new model showed a close agreement with the original model both in the qualitative behaviour of the ice cylinder and in the calculated force history. From this result it was concluded that the Carney ice material model was indeed suitable for use with a SPH approach.

This model was then used to simulate SHI impact on a modelled turbine blade leading edge, with geometry sourced from a major manufacturer and approximate material properties for wind turbine materials used. The impact scenario modelled was an impact, at right angles to the centre of the leading edge, of ice spheres of diameters 5,10 and $15 \mathrm{~mm}$ at velocities between 70 and $120 \mathrm{~m} \mathrm{~s}^{-1}$. From these simulations 
it was seen that the induced Von-Mises stress, while not sufficient to cause outright failure in the composite material layers, was large enough to cause delamination between the layers and damage to the outer, protective layers. In support of this, the erosion modelling function in LS-DYNA did indeed show erosion of the leading edge.

The aim of the modelling work presented in this paper is to further develop a realistic model of hailstone impact. The Tippmann experimental results will be used as a basis for modelling work, with the experimental setup being replicated within the initial simulation in order to provide a comparison between the simulated hailstone and experimental observation. The dependence of the physical behaviour on various simulation parameters will be explored.

\section{Material and Methods}

From the survey of the available literature on methods of modelling high-speed ice impacts, it can be seen that the current optimal approach is to use a Smooth Particle Hydrodynamic model with the strain-rate-sensitive material model as developed by Carney et al. [7] and thus it is the approach we will use as we look to extend these treatments further. The software package LS-DYNA [12] was chosen for this simulation work as it was the package used by the majority of the modelling literature, and its extensive use for crash simulation in the automotive industry suggests it to be suitable for modelling high-speed impacts with large deformations and high strain rates.

The Carney Ice material model is included in LS-DYNA as MAT_155 [13]. The hailstone model presented here uses this ice material model with the parameters used in the modelling work of Carney [7]. The ice sphere dimensions were chosen to replicate those used in the experimental work of Tippmann [6], and in particular an ice sphere diameter of $50.8 \mathrm{~mm}$ was chosen.

In order to replicate the experimental conditions, the impact target is modelled as an aluminium cylinder of the same dimensions as seen in the experiment, using a butterfly block mesh of approximately 10,000 solid elements. A simple elastic material model, using commonly available mechanical properties of aluminium, was chosen for the target cylinder since it is the physical behaviour of the ice sphere projectile that is of primary interest in these simulations. As a boundary condition, the bottom nodes of the solid block mesh were constrained on all three axes to simulate the target cylinder being held in place.

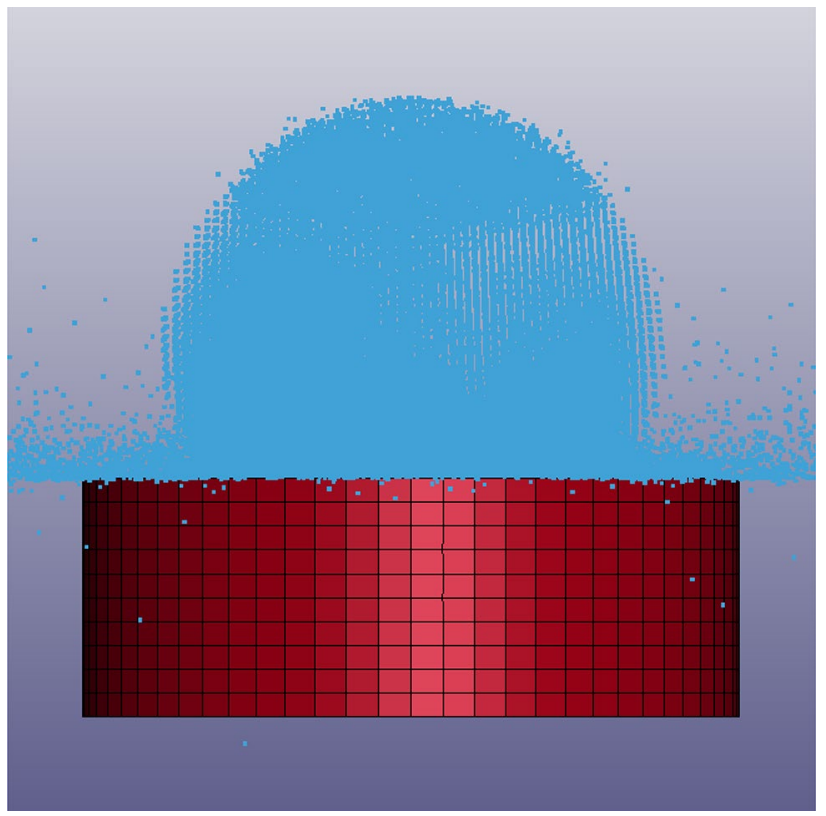

Fig. 1 A frame from the animation of the simulated impact

\section{Numerical Simulation}

A frame from an animation of a typical impact simulation is shown in Fig. 1, and the contact force over the time of the simulation is shown in Fig. 2. For this example, an impact velocity of $60.6 \mathrm{~m} / \mathrm{s}$ was chosen, as the experimental work features high-speed camera frames of an impact at that velocity and thus this scenario would be a useful point of comparison between the model and the experimental observations. It can be seen that the contact force quickly reaches a maximum approximately $0.1 \mathrm{~ms}$ after initial contact, reaching a peak force of approximately $22 \mathrm{kN}$, before quickly decaying to zero as the projectile rebounds. A simulation termination time of $0.6 \mathrm{~ms}$ was chosen to reduce computation complexity, as it can be seen that the primary physical behaviour of the projectile during the impact occurs during this time frame.

It was desirable to first determine the number of SPH nodes required to adequately simulate the ice projectile. To do this impact, simulations were carried out with multiple numbers of SPH nodes-from 50 nodes in each dimension up to 58 , representing a range of approximately $65,000-102,000 \mathrm{SPH}$ nodes. The force history for a selection of these simulations using different numbers of SPH nodes is plotted in Fig. 3. From this it can be seen that while there general behaviour of the projectile is consistent among these SPH meshes, the coarser SPH meshes give rise to anomalous sharp spikes in the contact force, particularly during the initial period of the impact. In the case of 58 nodes per dimension, however, these spikes have dissipated by the time the 


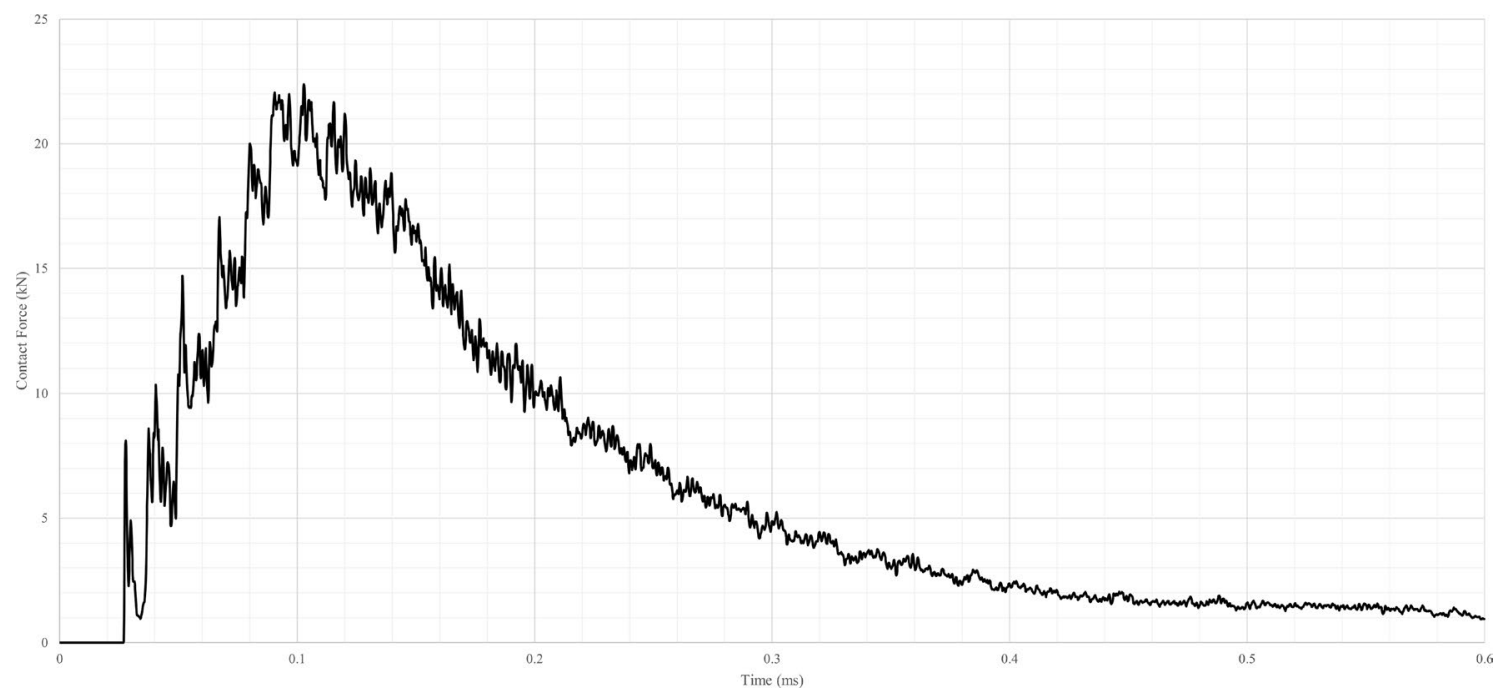

Fig. 2 A plot of the contact force between the ice sphere and the aluminium force transducer cap, over the timescale of the simulated impact

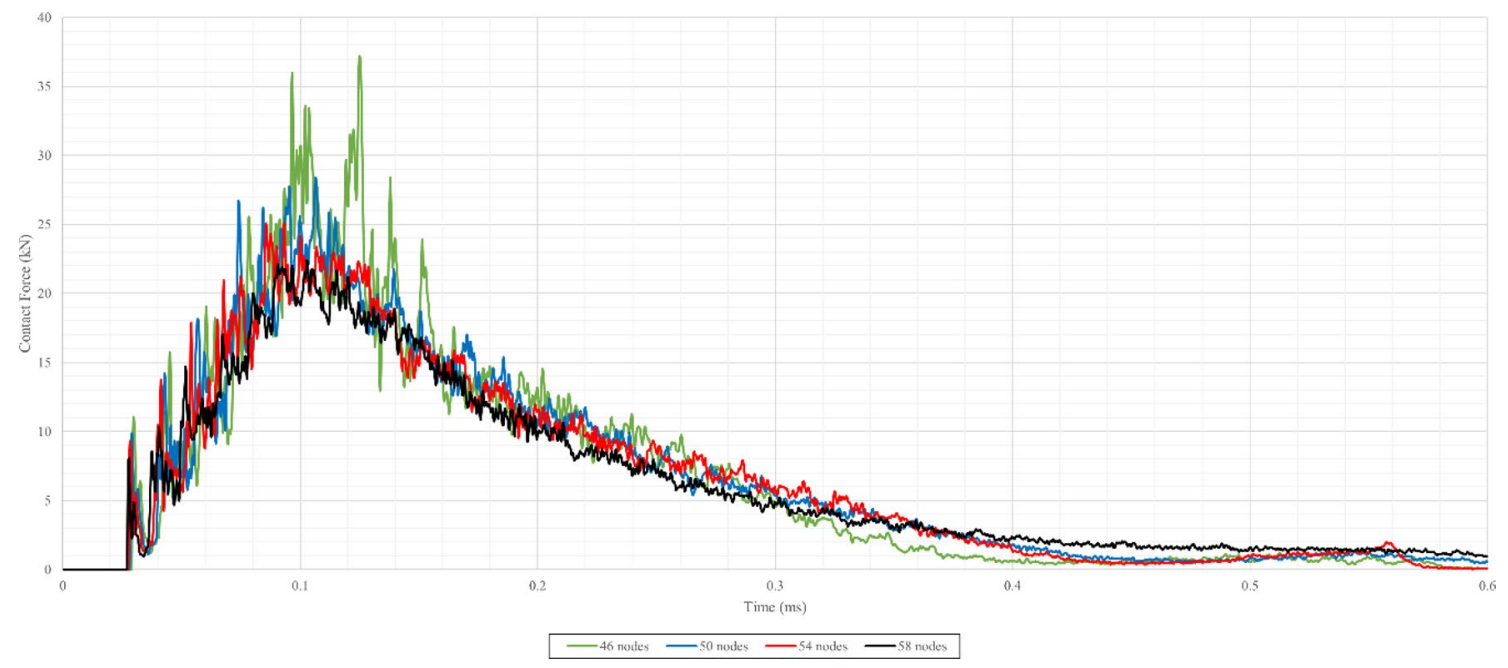

Fig. 3 A plot of the contact force histories for simulated impacts using three different numbers of SPH nodes

peak contact force is reached, and thus these peaks do not have a large effect on the value of the peak force observed in these simulations.

In order to further investigate the simulated projectile physics, simulations were run with varying values of the initial velocity. The peak forces observed in these simulations are plotted against impact velocity in Fig. 4, and against initial kinetic energy of the projectile in Fig. 5, in order to illustrate how the peak contact force changes as these parameters are varied.

\section{Experimental Validation}

While the presented simulations show a similar contact force profile over time to what is seen in the experimental result, the value of the peak contact force reached is higher in the simulated impact than the experiments, with the numerical model showing peak forces between $4 \%$ and $11 \%$ higher than what is expected at various values of initial velocity.

This discrepancy can be explained by comparing the physical behaviour of the projectile with the experimental high-speed footage shown in [6]. The footage shows extensive crack propagation and shattering in the ice sphere, with the projectile breaking up into many shards. This behaviour 


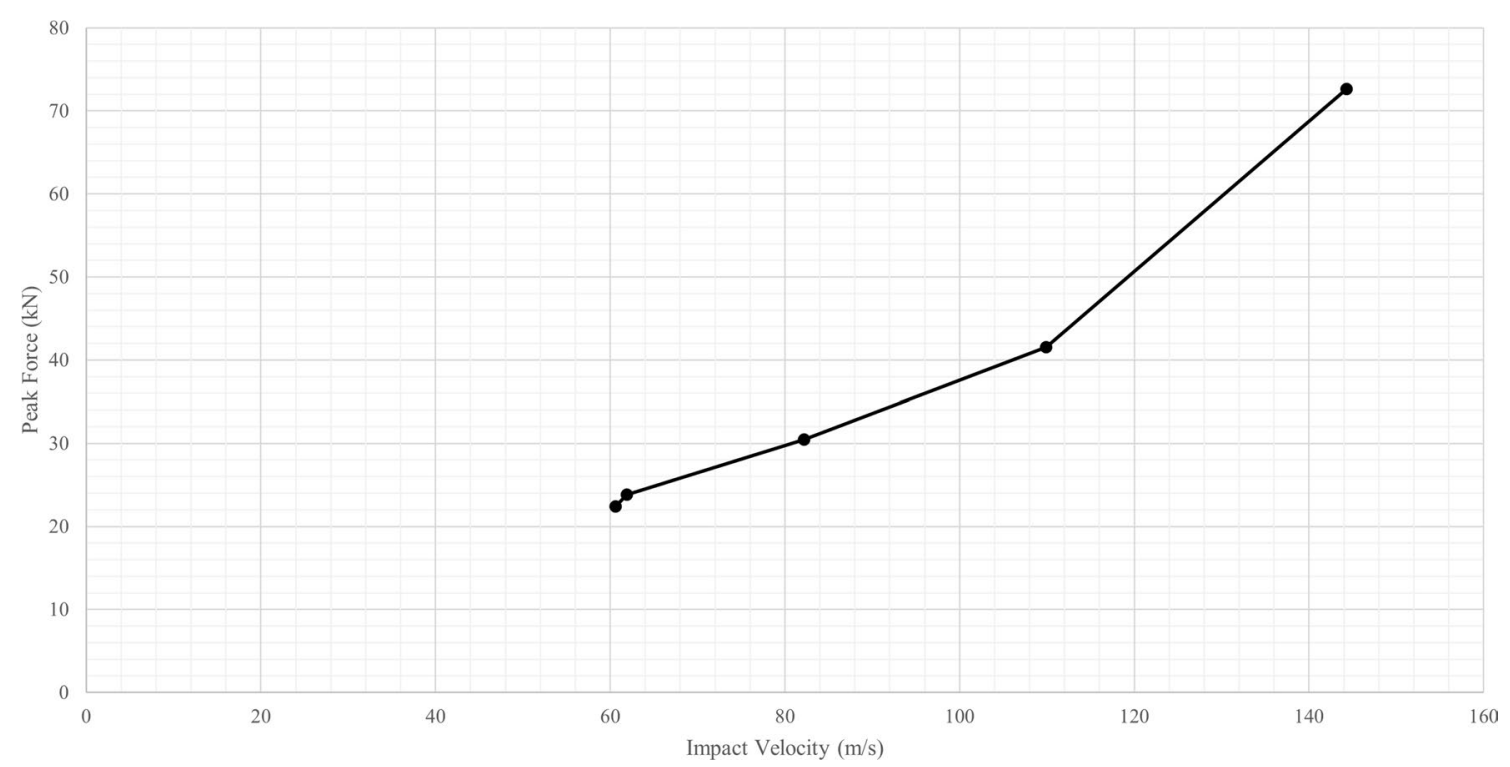

Fig. 4 A plot of peak impact force as a function of impact velocity from model simulations

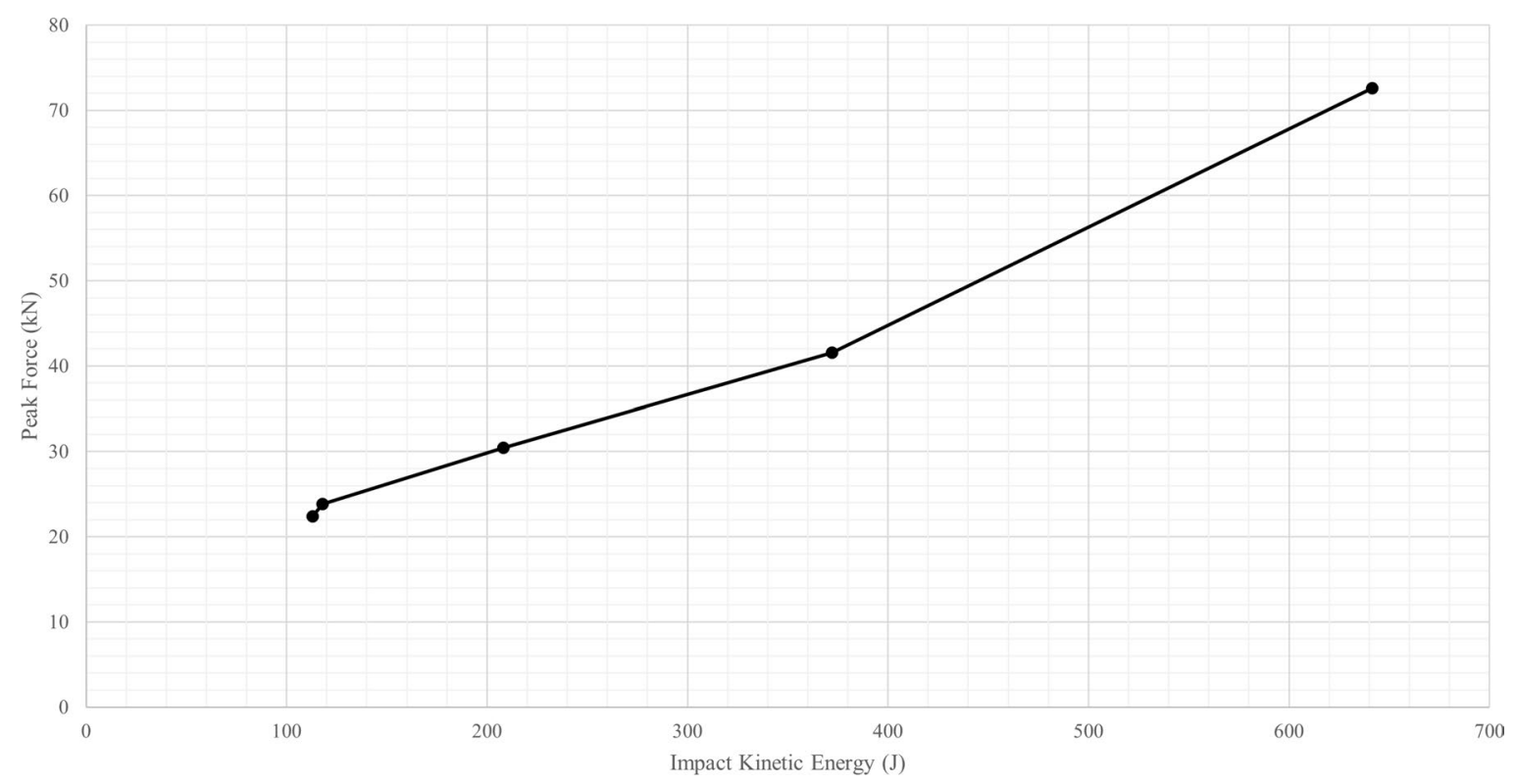

Fig. 5 A plot of peak impact force as a function of impact kinetic energy from model simulations

is not observed in the simulated projectile, in which material is ejected close to the impact site, but the main sphere remains largely intact. The reasons for this difference in behaviour are not well understood and will be investigated further. This difference in behaviour may influence the way in which the impact energy propagates through the projectile, and thus potentially explains the higher peak force seen in the simulation.

\section{Discussion}

For future work it important to find a modification to the current ice material model that more accurately shows the impact behaviour seen in the experimental literature, since this will affect the nature of the forces imparted onto the target and therefore on the predicted target material response. Once this is carried out and the model behaviour replicates the experimental results to an acceptable degree, 
the impact of an ice sphere on a composite target can then be considered.

\subsection{Application to Impact on Composites}

The LS-DYNA package is equipped with a model for composite material which includes the Chang-Chang model of damage in composites [14]. This theoretical model includes criteria for three different mechanisms of damage in a fibre-reinforced material: matrix cracking, compressive failure and fibre breakage. By monitoring the values of the expressions given in the criteria, the most likely mechanisms of damage for a composite material undergoing ice impact can be evaluated.

From the experimental and modelling literature, it is clear that hail ice has the potential to cause damage on the leading edge of wind turbine blades. However, due to the relative infrequency of hailstorm events, as well as the fewer number of hailstones per hailstorm event, the likelihood of significant fatigue-based erosion caused entirely by hail is smaller than that of rain, since the number of expected impacts and therefore load cycles is low. However, damage to the coating or composite material caused by ice impact, for example exposing fibres as the surface or causing delamination [15] of the composite layup beneath the surface, could provide a starting point for rain erosion, and hence a synergistic effect between the two erosion mechanisms could occur.

\subsection{Further Considerations}

Using the ice and composite materials models, the variability of damage sustained to various parameters can be investigated. For example, simulations of ice impacts involving different impact velocities, impact angles and hail diameters can be compared in order to investigate the dependence of the material response on these variables. For example, the majority of hail in the UK falls into the two smallest categories - those of diameter $5 \mathrm{~mm}$ or less and those of diameter between 5 and $9 \mathrm{~mm}$ [16]. Larger hail is more common elsewhere in the world [17]. It will also be important to explore how this is affected by the structure of hail ice projectiles, as real hail grows in onion-like layers spreading radially outwards from the core [18]. This knowledge, together with meteorological and turbine technical data, could be used to better predict the hail-based impact damage to the turbine leading edge expected at different geographical locations, and for different turbine blade lengths and tip speeds.

Another aspect that could be tackled using a modelling approach is the effect of bending loads on the simulated material damage during ice impact. Bending stresses have been shown experimentally to affect the rain erosion properties of glass fibre-reinforced composites [19], and so adding a constant load in order to simulate the stress caused by the blade rotational motion should be considered, as it may have a large effect on the material predicted response to solid particle impact. This effect will assume more significance in turbine blades with larger diameters, and so will become more important as turbine manufacturers seek to produce turbines with greater power output.

\section{Conclusions}

The previous work relating to the impact of ice on composite materials has been described, with the aim of adapting this work to predict erosion on the leading edge of wind turbines. The problem has been tackled from both an experimental and a modelling point of view

In the experimental literature, moderate damage was observed for few ice impacts. Later work yielded useful data relating to the impact mechanics of the ice sphere, which can be used to validate computational models.

The modelling literature has approached the hail ice model using a number of different numerical techniquesthese were outlined and evaluated, with the conclusion that Smooth Particle Hydrodynamics, together with the highly strain-rate dependent material model developed by Carney ([6]), was at this time the most advantageous method to employ during future modelling work.

These methods were implemented in LS-DYNA, but showed significant limitations when a simulation of an experimental procedure was compared to the real result, in that the modelled projectile did not show the same cracking and shattering behaviour that was present in the experimental high-speed footage. This indicates that additional modifications are required in order to adequately model the high-speed impact mechanics of an ice projectile.

Acknowledgements The authors would like to acknowledge the support of the Interreg (Northern Ireland-Ireland-Scotland) Special EU Programmes Grant No SPIRE2_INT-VA-049 "Storage Platform for the Integration of Renewable Energy (SPIRE 2)',.

\section{Compliance with ethical standards}

Conflict of interest On behalf of all authors, the corresponding author states that there is no conflict of interest.

Open Access This article is licensed under a Creative Commons Attribution 4.0 International License, which permits use, sharing, adaptation, distribution and reproduction in any medium or format, as long as you give appropriate credit to the original author(s) and the source, provide a link to the Creative Commons licence, and indicate if changes were made. The images or other third party material in this article are 
included in the article's Creative Commons licence, unless indicated otherwise in a credit line to the material. If material is not included in the article's Creative Commons licence and your intended use is not permitted by statutory regulation or exceeds the permitted use, you will need to obtain permission directly from the copyright holder. To view a copy of this licence, visit http://creativecommons.org/licenses/by/4.0/.

\section{References}

1. Keegan MH, Nash DH, Stack MM (2013) On erosion issues associated with the leading edge of wind turbine blades. J Phys D: Appl Phys. https://doi.org/10.1088/0022-3727/46/38/383001

2. Sareen A, Sapre CA, Selig MS (2014) Effects of leading edge erosion on wind turbine blade performance. Wind Energ 17:1531-1542

3. Sagol E, Reggio M, Ilinca A (2013) Issues concerning roughness on wind turbine blades. Renew Sustain Energy Rev 23:514-525

4. Chen J, Wang J, Ni AJ (2019) A review on rain erosion protection of wind turbine blades. J Coat Technol Res 16:15-24

5. Kim H, Kedward KT (2000) Modeling Hail Ice Impacts and Predicting Impact Damage Initiation in Composite Structures. AIAA J 38(7):1278-1288

6. Tippmann JD, Kim H, Rhymer JD (2013) Experimentally validated strain rate dependent material model for spherical ice impact simulation. Int J Impact Eng 57:43-54

7. Carney KS, Benson DJ, DuBois P, Lee R (2006) A phenomenological high strain rate model with failure for ice. Int J Solids Struct 43:7820-7839

8. Schulson EM (1997) The Brittle Failure of Ice under Compression. J Phys Chem B 101(32):6254-6258

9. Anghileri M, Castelletti L-ML, Invernizzi F, Mascheroni M (2005) A survey of numerical models for hail impact analysis using explicit finite element codes. Int J Impact Eng 31(8):929-944
10. Juntikka, R., and Olsson, R. (2009), "Experimental and Modelling Study of Hail Impact on Composite Plates", Proceedings of ICCM-17, Edinburgh, United Kingdom, July 2009.

11. Keegan MH, Nash D, Stack MM (2013) Numerical modelling of hailstone impact on the leading edge of a wind turbine blade. EWEA Annual Wind Energy Event, Vienna, Austria

12. LS-DYNA User's Manual, Livermore Software Technology Corporation

13. LS-DYNA theory manual, Livermore Software Technology Corporation

14. Chang F-K, Chang K-Y (1987) A Progressive Damage Model for Laminated Composites Containing Stress Concentrations. J Compos Mater 21(9):834-855

15. Olsson R, Donadon MV, Falzon BG (2006) Delamination threshold load for dynamic impact on plates. Int J Solids Struct 43(10):3124-3141

16. Macdonald H, Infield D, Nash DH, Stack MM (2016) Mapping hail meteorological observations for prediction of erosion in wind turbines. Wind Energ 19:777-784

17. Xie B, Zhang Q, Wang Y (2010) Observed Characteristics of Hail Size in Four Regions in China during 1980-2005. J Clim 23(18):4973-4982

18. List R (2014) New hailstone physics. Part I: Heat and mass transfer (HMT) and growth. J Atmos Sci 71:1508-1520

19. Pugh K, Rasool G, Stack MM (2019) Raindrop Erosion of Composite Materials: Some Views on the Effect of Bending Stress on Erosion Mechanisms. J Bio Tribo Corros. https://doi.org/10.1007/ s40735-019-0234-8

Publisher's Note Springer Nature remains neutral with regard to jurisdictional claims in published maps and institutional affiliations. 